\title{
Tecniche chirurgiche dei tessuti tendo-legamentosi
}

\author{
Nicola Maffulli ${ }^{1,2, a}(\bowtie)$, Francesco Oliva ${ }^{3, b}$ \\ ${ }^{1}$ Department Centre for Sports and Exercise Medicine, Queen Mary University of London, Barts and The London \\ School of Medicine and Dentistry, Mile End Hospital, London, UK \\ ${ }^{2}$ Dipartimento di Medicina Fisica e Riabilitativa Università degli Studi di Salerno, Facoltà di Medicina e Chirurgia, \\ Azienda Ospedaliera San Giovanni Di Dio e Ruggi d'Aragona, Salerno, Italia \\ ${ }^{3}$ Dipartimento di Ortopedia e Traumatologia, Università degli Studi di Roma "Tor Vergata", Facoltà di Medicina \\ e Chirurgia, Roma, Italia \\ an.maffulli@qmul.ac.uk; bolivafrancesco@hotmail.com
}

Pubblicato online: 13 aprile 2013

(c) Springer-Verlag Italia 2013

È stata una vera gioia accettare di organizzare questo volume di Lo Scalpello su un argomento cui siamo visceralmente legati da motivi di ricerca e passione.

L'ortopedia da molti anni ha sviluppato un approccio più scientifico nei confronti di tessuti in passato trascurati, quali i muscoli, i tendini e i legamenti. L'unità motoria, quando è approcciata chirurgicamente, necessita di un approfondimento culturale sulla componente dei tessuti molli diverso dal passato.

Biologicamente, l'osso ha maggiori capacità e probabilità di guarire rispetto a muscoli, legamenti e tendini: quindi, da un punto di vista chirurgico, abbiamo bisogno di un continuo miglioramento delle tecniche stesse.

Oggi ci troviamo nell'era della scienza di base applicata all'ortopedia, e dobbiamo aggiornare il nostro pensiero in base ai progressi scientifici. In quest'ottica è stata fondata due anni fa la prima Società scientifica al mondo che si occupa dei tessuti molli, la Italian Society of Muscles, Ligaments and Tendons (ISMULT) (www.ismult.com). Il carattere traslazionale di questa iniziativa consente a diversi compartimenti biologici e medici di incontrarsi in una sede comune e finalmente aprire il confronto sulle diverse esigenze.

In ultimo, è con grande entusiasmo che vorremmo ringraziare il presidente dell'OTODI, il Prof. Carlo De Roberto, per averci invitato a realizzare questo volume e per aver dimostrato un grande interesse scientifico nei confronti delle nostre attività: è stato un onore prenderci carico di questa iniziativa.

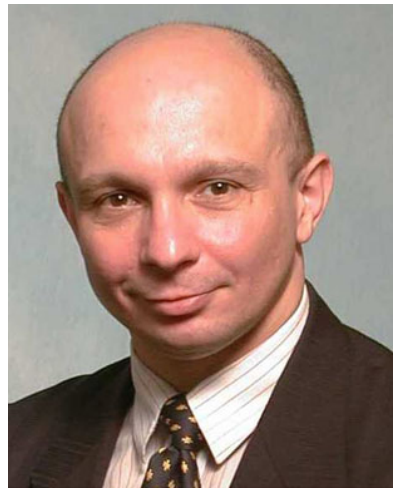

Nicola Maffulli

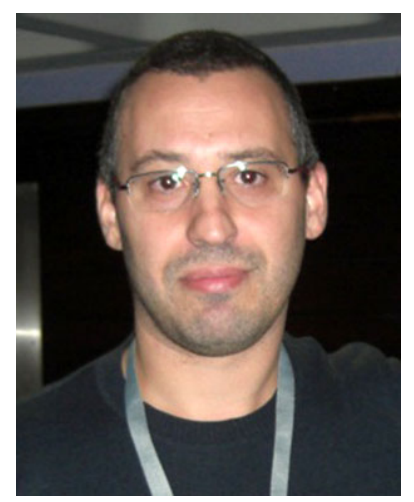

Francesco Oliva 